\title{
A Manipulated Deformable Object as an Underactuated Mechanical System
}

\author{
Herbert G. Tanner and Kostas J. Kyriakopoulos
}

\begin{abstract}
Deformable objects under manipulation can be modeled using finite elements. The resulting model is in fact an underactuated mechanical system. The consequences of any type of constraints revealed by the modeling procedure is explored. The study of deformable object models within the framework of underactuated mechanical systems indicated the existence of second order nonholonomic constraints. For the identification of this kind of constraints, the authors have developed computationally efficient and significantly simpler mathematical tools. Their methodology is illustrated and tested by an example.
\end{abstract}

\section{Introduction}

In robot manipulation the object that was being handled was traditionally considered rigid. This assumption on the object's nature simplified significantly the problem. The approach was justified from nearly every point of view. It allowed researchers to focus on the new system being developed, namely the robot, and adjusted its environment to facilitate analysis: no obstacles present, clean and absolutely known environment, rigid objects.

As problems involved in robot manipulation find their solution with time and great effort by the robotics community, all of these assumptions are gradually relieved. Obstacle avoidance has been considered, adaptation methods explored, sensor information taken into account. It is about time we did something about the assumption concerning the object being rigid. 
In what follows we shall discuss some properties of a model obtained for deformable objects being manipulated by robotic arms. The motivation for investigating deformable materials came from the study of a multiple mobile manipulator system which handles a deformable object [1]. When focusing on the object issue, we first had to choose an appropriate model to describe its behavior.

Previous approaches to deformable object handling focused mainly on formulating general continuous dynamic equations for the object. The aim was to construct an appropriate model to enhance computation or allow for the application of certain control strategies. Sun et al. [2] followed Terzopoulos' [3] hybrid approach to deformable objects. This approach originated from the study of Computer Aided Design and computer graphics. It is characterized by the decomposition of the deformable object to a reference component and a deformation component. The former represents the original shape of the object and the latter the change in its shape as a result of the applied load. The position of any point in the body can be then determined by a superposition of the two components.

Kosuge et al. [4] used finite elements. Loading conditions included only bending of a sheet metal and examined the problem of controlling the static deformation of the plate when handled by a dual manipulation system. The static assumption simplifies considerably the dynamics of the object.

$\mathrm{Wu}$ et al. [5] pointed out that a flexible object is actually a distributed parameter system and approximated it by considering a lumped parameter model. They also assumed, quite reasonably, that the object is not very soft which means that it undergoes only small deflections during its interaction with the robot. A case of a flexible metal plate is considered. To construct the lumped parameter system they exploited the geometry of the system of the robot grasping the metal plate. It is not clear, however, how this can be extended to the case of other objects with arbitrary shape.

Yukawa et al. [6] investigated a vibrating flexible object and modeled it using model reduction theory. The aim was to realize position control while suppressing the vibration of the object. Vibration is assumed to take place in a two dimensional space. The modeling approach for the object begins with the distributed parameter model and ends up with a finite dimensional model.

In our approach to modeling deformable objects we decided to use finite elements based on the elastodynamic equations, motivated mainly by an engineering attitude towards the problem in hand. This step has a decisive impact on the nature of the problem we would then have to face. A question arises concerning the structural properties of the model thus obtained; how does that behave from the control point of view; what are the limitations and what can one hope to achieve.

Talking about achievement, we would better determine what needs to be done first. In our study of the multiple mobile manipulator system we placed emphasis on the deformable object, since this defines at a great degree the whole system's specifications and requirements. Everything has to be done with an eye on the object: should it be glass it must not break, should it be paper, cloth or leather it must not tear apart, should it be wood it must not fracture, should it be a metal plate it must not bend too much, and so on. We therefore needed to plan the motion of the object 
so that it can be safely been transferred from one point to another, without placing excessive load on the robots which could tip over or reach a singular position in their effort to accomplish their mission. That is why we had to see what the model we chose for the object could do.

First, we identified an underactuated system [7]. Finite element procedures result to a model which has a few points, or nodes, where load is directly exerted and a number of intermediate points which are more or less influenced by that load. Seen from the control perspective, the nodes where load is applied are directly actuated while the rest are statically and dynamically coupled to them. Therefore, there is a number of degrees of freedom which are actuated and others which are not. This is roughly the idea of an underactuated system: underactuated systems have fewer control inputs than degrees of freedom.

Viewing the manipulated object as an underactuated system we can considerably broaden the notion of a "deformable object" to the case of systems which are not continuous: chains, structures with passive joints, flexible links, rolling contacts, etc. This allows a generalization of our approach to object handling and puts a great variety of systems in a unified framework of study.

In the analysis of underactuated systems, it will soon become clear that a part of their dynamics, (which others prefer to call the zero dynamics), can be thought of as a set of dynamic constraints. In this context we refer to these equations as intrinsic constraints, since they stem directly from the nature of the system and are present regardless of the assumptions one may make for the manipulated object. Apart from these, practice can enforce other limitations such as the avoidance of fracture or excessive loading which can result to plastic (non-elastic) deformation. These external limitations can relate to material strength limitations, conditions for obstacle avoidance during the motion of the object, and so forth. Normally, the external constraints can be expressed in the form of a set of inequalities.

\subsection{The Underactuated System}

Strictly speaking, a deformable object has an infinite number of degrees of freedom. This is because every point in the body can be considered as an individual degree of freedom. The deeper one goes in a microscopic scale, the bigger the number grows. Although mathematicians would feel quite comfortable with the notion of infinity in the problem, this would probably distress most engineers who are assigned to the task of proposing a realistic solution.

Engineers would therefore try to decrease this number to a finite one and make a tradeoff between computational complexity and accuracy of solution. In the process of this simplification in order to deal with the problem using existing well known and approved tools, a natural approach is to "discretize" the object: consider it as a system of identical interconnected material elements that each contributes by a small portion to the system's overall behavior. This is actually the idea underlying the finite elements approach.

The extend of discretization depends on many factors. One is the individual characteristics of the material: objects that are fairly rigid do not require much dis- 
cretization; flexible objects need many elements to sufficiently describe their shape. Another factor is loading conditions: for the same object, if a large load is to be applied the discretization grid should be denser since each element would be called to undertake a larger displacement. If the measure of this displacement is excessive, then the assumptions on which the approximation is based could cease to hold and the results may not be valid any more. For a given material and approximately known loading conditions, a pretty good discretization grid can be constructed.

The finite element method yields a dynamic model in which some degrees of freedom are driven directly as a result of external load and the rest comply with the displacement of the former. The degrees of freedom that are directly controlled correspond to the nodes of the grid at the location where the manipulators grasp the object. All other nodes are displaced in accordance to the loading conditions imposed by the driven nodes. The whole system can be therefore classified as underactuated [8] since the number of degrees of freedom being directly controlled is less than the total number of degrees of freedom in the system.

The class of underactuated mechanical systems is very broad. It includes systems with passive joints, flexible link constructions, chain mechanisms, mobile robots, and many others. These systems are peculiar in the sense that their Lagrangian dynamics may contain undesirable properties such as non-minimum phase zero dynamics, nonholonomic constraints, etc. Viewing the manipulated object as an underactuated system allows for the investigation of new manipulation tasks, including systems of rigid bodies or combinations of rigid and deformable objects. The object being handled can now be a truss, a frame, a plate, a chain or an arbitrary shaped three dimensional body and the material considered can be practically anything that humans themselves can manipulate.

In Lagrangian dynamics the equations of motion for a mechanical system with $n$ degrees of freedom can be derived as

$$
\frac{d}{d t}\left(\frac{\partial L}{\partial \dot{q}_{i}}\right)-\frac{\partial L}{\partial q_{i}}=\mathbf{F}_{i} \quad i=1, \ldots, n,
$$

where $q$ are the generalized coordinates chosen for the system:

$$
\mathbf{q}=\left(\begin{array}{lll}
q_{1} & \cdots & q_{n}
\end{array}\right)^{T} \in \mathbf{Q} \subset \mathfrak{R}^{n}
$$

It is well known that these equations can take the matrix equation form:

$$
\mathbf{M}(\mathbf{q}) \ddot{\mathbf{q}}+\mathbf{C}(\mathbf{q}, \dot{\mathbf{q}}) \dot{\mathbf{q}}+\mathbf{K}(\mathbf{q})=\mathbf{B}(\mathbf{q}) \mathbf{u}
$$

where $\mathbf{M}$ is the inertia matrix, which is symmetric and positive definite, $\mathbf{C}$ is a matrix related to Coriolis and centrifugal forces, $\mathbf{K}$ is the vector formed by the elastic and gravitational terms and $\mathbf{u}$ is the input to the system. For the case of underactuated systems, $\mathbf{u} \in \mathfrak{R}^{m}$, with $m<n$.

The different dimension between the space of control inputs and the space of generalized coordinates motivates the partition of the latter into an actuated and an unactuated subspace. Without loss of generality we can rearrange the terms in the $q$ vector as follows:

$$
\mathbf{q}^{T}=\left(\begin{array}{ll}
\mathbf{q}_{1}{ }^{T} & \mathbf{q}_{2}{ }^{T}
\end{array}\right)
$$


where $\mathbf{q}_{1} \in \mathbf{Q}_{1} \subset \mathfrak{R}^{n-m}$ corresponds to the unactuated coordinates and $\mathbf{q}_{2} \in \mathbf{Q}_{2} \subset \mathfrak{R}^{m}$ to the actuated or controlled coordinates. In the light of the above partition, equation (1) can be written [8]

$$
\begin{aligned}
& \mathbf{m}_{11} \ddot{\mathbf{q}}_{1}+\mathbf{m}_{12} \ddot{\mathbf{q}}_{2}+\mathbf{c}_{1}(\mathbf{q}, \dot{\mathbf{q}})+\mathbf{k}_{1}(\mathbf{q})=\mathbf{0} \\
& \mathbf{m}_{21} \ddot{\mathbf{q}}_{1}+\mathbf{m}_{22} \ddot{\mathbf{q}}_{2}+\mathbf{c}_{2}(\mathbf{q}, \dot{\mathbf{q}})+\mathbf{k}_{2}(\mathbf{q})=\mathbf{b}(\mathbf{q}) \mathbf{u}
\end{aligned}
$$

where the matrix $\mathbf{b}(\mathbf{q}) \in \mathfrak{R}^{m \times m}$ is assumed nonsingular.

Equation (2a) is a set of $n-m$ second order uncontrolled differential equations which can be considered as dynamic constraints. Some prefer to interpret these equations as the zero dynamics, however this is not formally correct since no output has been specified for this system. In this context we will name these equations "intrinsic constraints" to distinguish them from any external imposed constraints that can relate to material strength limitations or obstacle avoidance requirements.

A natural question to ask is what kind of constraints these are. Can these differential equations be integrated once? If so, then one has a set of expressions relating the generalized velocities with the generalized coordinates, i. e. there will be no acceleration terms present. Do these resulting equations permit a second final integration? Should such integration be permitted, the result would be a set of algebraic equations relating the generalized coordinates.

In this final case, things are rather simple: The algebraic equations thus obtained define an $n-m$ hypersurface on which the system must live. This is the case of holonomic constraints. For an object being manipulated, this means that the robots have no control whatsoever over $n-m$ degrees of freedom of the system. These degrees are completely specified by the remaining $m$. Therefore, they cease to be degrees of freedom: they are just some fully dependent variables that can be equally ignored in the dynamic model. This could indicate a rigid behavior exhibited by a part of the object. In fact, such a situation is improbable, since even "rigid" material exhibits some sort of elastic behavior, at least in theory. It may arise however if during the initial modeling procedure some physical constraints have been overlooked, and the model comes later on to bring them into evidence. This implies that there is a way to improve the model and reduce the complexity of the problem.

If the equations can be integrated only once, one ends up with a set of first order nonholonomic constraints. There are only generalized coordinates and their first order time derivatives present. Now things are more complicated: these relations imply that there are limitations in the space of velocities that do not influence directly the space of positions. The influence of these velocity limitations is quite subtle. They impose significant limitations in control. In simple terms they imply that one may be able to steer the system from one place to another but the path it must follow is not necessarily the shorter in some respect. A simple example is parking a car: there is a steering sequence you have to follow - one cannot simply turn the car in any desirable direction. If this is the case, then for the task of planning trajectories for the object one has to resort to special and heavy mathematical tools. 
The last case is when the equations cannot be integrated at all. This implies the presence of second order nonholonomic constraints. The deeper impact of such constraints has not been very well understood yet. Such systems could be hard to control, as in the case of first order nonholonomic constraints, but sometimes the systems can even be controlled with linear time-invariant controllers [8]. This is typical for systems that have an elastic/gravity (potential) term. The underlying characteristics of second order nonholonomic constraints will be clarified as research continues.

It is therefore important to investigate the type of the constraints imposed on the system, since these could determine to a certain degree the controllability properties of the system, or imply ways in which the model can be improved.

\section{Collocated Linearization}

Certain forms of system description enhance analysis. A notable one is the normal form, which will be used in subsequent analysis. This form reveals a structure that is particularly useful in identifying the type of constraints imposed on the system. Many of the previous results concerning nonholonomic constraints have used this type of description.

The normal form is obtained by a standard technique of feedback linearization. The system ( $2 a-2 b)$ can always be partially feedback linearized with respect to the actuated degrees of freedom. This is an important property of such systems [8]. The process of partial feedback linearization for this class of systems is also known as collocated linearization.

It can easily be understood that the matrix $\mathbf{m}_{11}$ in equation (2a) is square and nonsingular. The latter is guaranteed by the positive definiteness of the inertia matrix M. If (2a) is solved for $\ddot{\mathbf{q}}_{1}$, we obtain

$$
\ddot{\mathbf{q}}_{1}=-\mathbf{m}_{11}^{-1}\left[\mathbf{m}_{12} \ddot{\mathbf{q}}_{2}+\mathbf{c}_{1}(\mathbf{q}, \dot{\mathbf{q}})+\mathbf{k}_{1}(\mathbf{q})\right]
$$

This can be used to substitute $\ddot{\mathbf{q}}_{1}$ in $(2 \mathrm{~b})$ so that it becomes:

$$
\overline{\mathbf{m}}(\mathbf{q}) \ddot{\mathbf{q}}_{2}+\overline{\mathbf{c}}(\mathbf{q}, \dot{\mathbf{q}})+\overline{\mathbf{k}}(\mathbf{q})=\mathbf{b}(\mathbf{q}) \mathbf{u}
$$

where

$$
\begin{aligned}
& \overline{\mathbf{m}}(\mathbf{q})=\mathbf{m}_{22}(\mathbf{q})-\mathbf{m}_{21}(\mathbf{q}) \mathbf{m}_{11}{ }^{-1}(\mathbf{q}) \mathbf{m}_{12}(\mathbf{q}) \\
& \overline{\mathbf{c}}(\mathbf{q}, \dot{\mathbf{q}})=\mathbf{c}_{2}(\mathbf{q}, \dot{\mathbf{q}})-\mathbf{m}_{21}(\mathbf{q}) \mathbf{m}_{11}{ }^{-1}(\mathbf{q}) \mathbf{c}_{1}(\mathbf{q}, \dot{\mathbf{q}}) \\
& \overline{\mathbf{k}}(\mathbf{q})=\mathbf{k}_{2}(\mathbf{q})-\mathbf{m}_{21}(\mathbf{q}) \mathbf{m}_{11}{ }^{-1}(\mathbf{q}) \mathbf{k}_{1}(\mathbf{q})
\end{aligned}
$$

Using now the linearizing feedback

$$
\mathbf{u}=\mathbf{b}(\mathbf{q})^{-1}[\overline{\mathbf{m}}(\mathbf{q}) \mathbf{v}+\overline{\mathbf{c}}(\mathbf{q}, \dot{\mathbf{q}})+\overline{\mathbf{k}}(\mathbf{q})]
$$

where $v$ is the new control vector, the system $(2 a-2 b)$ can take the form

$$
\ddot{\mathbf{q}}_{2}=\mathbf{v}
$$


where the terms are defined as follows

$$
\ddot{\mathbf{q}}_{1}=\mathbf{J}(\mathbf{q}) \ddot{\mathbf{q}}_{2}+\mathbf{R}(\mathbf{q}, \dot{\mathbf{q}})
$$

$$
\begin{aligned}
& \mathbf{J}(\mathbf{q})=-\mathbf{m}_{11}{ }^{-1}(\mathbf{q}) \mathbf{m}_{12}(\mathbf{q}) \\
& \mathbf{R}(\mathbf{q}, \dot{\mathbf{q}})=-\mathbf{m}_{11}{ }^{-1}(\mathbf{q}) \mathbf{c}_{1}(\mathbf{q}, \dot{\mathbf{q}})-\mathbf{m}_{11}{ }^{-1}(\mathbf{q}) \mathbf{k}_{1}(\mathbf{q})
\end{aligned}
$$

\subsection{State Space Description}

The state space equations of the underactuated system can be easily obtained by equations (4) by setting:

$$
\mathbf{x}_{1}=\mathbf{q}_{1} \in \mathfrak{R}^{n-m}, \quad \mathbf{x}_{2}=\mathbf{q}_{2} \in \mathfrak{R}^{m}, \mathbf{x}_{3}=\dot{\mathbf{q}}_{1}, \quad \mathbf{x}_{4}=\dot{\mathbf{q}}_{2}
$$

This way, equations (4) can take the form:

$$
\begin{aligned}
\dot{\mathbf{x}}_{1} & =\mathbf{x}_{3} \\
\dot{\mathbf{x}}_{2} & =\mathbf{x}_{4} \\
\dot{\mathbf{x}}_{3} & =\mathbf{J}\left(\mathbf{x}_{1}, \mathbf{x}_{2}\right) \mathbf{v}+\mathbf{R}\left(\mathbf{x}_{1}, \mathbf{x}_{2}, \mathbf{x}_{3}, \mathbf{x}_{4}\right) \\
\dot{\mathbf{x}}_{4} & =\mathbf{v}
\end{aligned}
$$

with configuration vector $\mathbf{x}=\left[\begin{array}{ll}\mathbf{x}_{1} & \mathbf{x}_{2}\end{array}\right]^{T} \in \mathbf{M} \subset \mathfrak{R}^{n}$. The above equations have an unactuated linear part $(5 \mathrm{a}-5 \mathrm{~b})$ and an actuated nonlinear part $(5 \mathrm{c}-5 \mathrm{~d})$. They will be the starting point for the analysis of the constraint equations and the stabilization properties that will follow. We chose to rename the configuration variables because the partition of the original configuration space and the rearrangement of variables can easily cause confusion.

\section{Constraint Classification}

\subsection{Preliminaries}

In the sequel we use some mathematical concepts from the field of differential geometry. For the reader who is not familiar we these terms we will attempt a short and informal introduction. Those familiar with the terms may skip this section. The definitions given informally below are by no means complete nor acceptable mathematically and they serve only to allow a non-specialist to follow intuitively our approach. The interested reader can refer to [9].

A manifold is a locally Euclidean space. Locally means that one cannot use the same constructions (not even the same coordinate system) to move from a point to any other point. A sphere is a manifold which is only locally Euclidean. We consider the earth's surface as a plane because in our scale it seems so, and practically it 
is a excellent approximation. Everyone however has a clear picture of the spherical shape of Earth and does not expect to join the north and the south pole with a straight line without that line crossing the surface. The planar approximation can only hold locally.

A tangent vector is a vector tangent to the manifold's surface attached to a point on the manifold. The tangent vector is always referred to in connection to the point at which it is attached. All tangent vectors at a point form the tangent space at that point. A vector field is a mapping that assigns to each point on the manifold, a tangent vector on that manifold. The velocity of a particle moving on the manifold is a vector field. Vector fields are closely related to differentiation. That is why we use the symbols $\partial / \partial x$ to denote the base vectors in the tangent space. The right hand side of the state equations of the system can define a set of vector fields $\mathbf{g}_{i}(\mathbf{x})$ :

$$
\dot{\mathbf{x}}=\mathbf{g}_{1}(\mathbf{x}) v_{1}+\cdots+\mathbf{g}_{p}(\mathbf{x}) v_{p}
$$

Having one or more vector fields on a manifold you can assign at each point one or more tangent vectors. These tangent vector may span a tangent subspace at this point. The collection of all tangent subspaces generated by the tangent vectors of the vector fields forms a distribution.

Several operations can be defined on vector fields. One of them is the Lie bracket which resembles an outer product operation. The product of the Lie bracket operation between two vector fields is another vector field. Under the Lie bracket operations the vector fields can form an algebra, namely the Lie algebra. With every distribution spanned by some vector fields an algebra is associated. Studying the mathematical properties of this algebra one can draw significant conclusions concerning the controllability properties of a system the vector fields of which generate the associated distribution.

\subsection{Definitions and Constraint Identification}

Nonholonomic constraints can be identified with the use of some tools from differential geometry. Consider equation (5). We state the following definition, adopted from [10]

Definition 1 [10]: Consider the system (5) and define the following vector fields:

$$
\begin{aligned}
& \tau_{0}=\sum_{j=1}^{m} \dot{x}_{2, j} \frac{\partial}{\partial \dot{x}_{2, j}}+\sum_{k-1}^{n-m}\left(\dot{x}_{1, k} \frac{\partial}{\partial x_{1, k}}+R_{k} \frac{\partial}{\partial \dot{x}_{1, k}}\right)+\frac{\partial}{\partial t}, \\
& \tau_{j}=\frac{\partial}{\partial \dot{x}_{2, j}}+\sum_{i=1}^{n-m} J_{i j} \frac{\partial}{\partial \dot{x}_{1, i}}, \quad j=1, \ldots, m
\end{aligned}
$$

and let $\Delta=\operatorname{span}\left\{\tau_{0}, \tau_{j}\right\}$ be the distribution generated by them. Consider the accessibility algebra $\tilde{C}$ of the distribution $\Delta$, i. e. the smallest subalgebra that 
contains the vector fields $\left\{\tau_{0}, \tau_{j}\right\}$. Let $\widetilde{\mathbf{C}}$ be the accessibility distribution generated by the accessibility algebra. If $\operatorname{dim} \widetilde{\mathbf{C}}(\mathbf{x}, t)=2 n+1 \quad \forall(\mathbf{x}, t) \in \mathbf{M} \times \mathfrak{R}$, then the system (5) is called completely second order nonholonomic.

When the condition for the dimension of the accessibility algebra is satisfied for the underactuated system (5), then it possesses second order nonholonomic constraints. This implies that the constraint equations ( $2 b)$ cannot be integrated to produce a distribution for the system. One should carefully distinguish between the integration with respect to time and the integration we are discussing. The latter refers to Frobenius integration, i. e. finding smooth functions $\lambda_{i}$ that solve a partial differential equation $\frac{\partial \lambda_{i}}{\partial\left[\begin{array}{ll}\mathbf{x} & \dot{\mathbf{x}}\end{array}\right]}\left[\begin{array}{ll}\tau_{0} & \tau_{j}\end{array}\right] \equiv \mathbf{0}$. If such an integration is possible then it means that the dynamic equations restrict the system to develop on an $n+m$ dimensional distribution. If on the other hand, the constraints are second order nonholonomic, the dimension of the state space remains $2 n$. Whether these constraints influence decisively the controllability properties of the system is another issue which will be discussed later.

Suppose now that the constraints are not second order nonholonomic and are integrated once. The resulting equations will now relate the generalized coordinates with their first time derivatives. The resulting equations can then be expressed in the form:

$$
\mathbf{A}(\mathbf{q}) \dot{\mathbf{q}}=\mathbf{0}, \quad \mathbf{A} \in \Re^{(\mathbf{n}-\mathbf{m}) \times \mathbf{n}}
$$

The annihilators of the rows of matrix $\mathbf{A}(\mathbf{q})$ form another matrix, $\mathbf{S}(\mathbf{q}) \in \mathfrak{R}^{m \times n}$, for which

$$
\mathbf{A}(\mathbf{q}) \mathbf{S}(\mathbf{q}) \equiv \mathbf{0}
$$

The existence of $\mathbf{S}(\mathbf{q})$ implies a relationship of the form [11]

$$
\dot{\mathbf{q}}=\mathbf{S}^{T}(\mathbf{q}) \boldsymbol{\eta}, \quad \boldsymbol{\eta} \in \mathfrak{R}^{m}
$$

The columns of $\mathbf{S}^{T}(\mathbf{q})$ are vector fields which define a distribution. Consider the accessibility algebra that is generated by those vector fields (the smaller subalgebra that contains those vector fields). If the accessibility algebra has a dimension equal to $n$, then the system is completely first order nonholonomic by Frobenius theorem. This means that the system is restricted to evolve on a tangent bundle of dimension $n+m$ which can be readily shown with the standard analysis that will follow. Nonholonomic constraints allow the system to reach any desired position, however they locally limit the directions on which the system can move. The limitation does not apply to configuration variables but rather to velocities: at a specific configuration the system may not be able to develop velocity at certain directions. The number $n+m$ can be thought of as the new dimension of the state space.

After differentiation, equation (7) becomes

$$
\ddot{\mathbf{q}}=\mathbf{S}^{T}(\mathbf{q}) \dot{\boldsymbol{\eta}}+\dot{\mathbf{S}}(\mathbf{q}) \boldsymbol{\eta}
$$

This can be substituted in (1) to yield 


$$
\mathbf{M}(\mathbf{q})\left(\mathbf{S}^{T}(\mathbf{q}) \dot{\eta}+\dot{\mathbf{S}}(\mathbf{q}) \eta\right)+\mathbf{C}(\mathbf{q}, \dot{\mathbf{q}}) \dot{\mathbf{q}}+\mathbf{K}(\mathbf{q})=\mathbf{B}(\mathbf{q}) \mathbf{u}
$$

and multiplying by $\mathbf{S}(\mathbf{q})$ from the right

$$
\mathbf{S} \mathbf{M} \mathbf{S}^{T} \dot{\boldsymbol{\eta}}+\left\{\mathbf{S} \mathbf{M} \dot{\mathbf{S}} \boldsymbol{\eta}+\mathbf{S C}\left(\mathbf{q}, \mathbf{S}^{\mathbf{T}} \boldsymbol{\eta}\right) \mathbf{S}^{T} \boldsymbol{\eta}\right\}+\mathbf{S} \mathbf{K}=\mathbf{S} \mathbf{B} \mathbf{u}
$$

where the dependence on $\mathbf{q}$ has been dropped.

Equation (8) is now $m$ dimensional and give the reduced dynamic model of the system (1). After some algebraic manipulation and rearranging of terms, equation (8) can be written as

$$
\dot{\boldsymbol{\eta}}=\mathbf{D}(\mathbf{q})+\mathbf{G}(\mathbf{q}, \boldsymbol{\eta}) \mathbf{u}
$$

and form the new reduced order model of the system

$$
\begin{aligned}
& \dot{\mathbf{q}}=\mathbf{S}^{T} \boldsymbol{\eta} \\
& \dot{\boldsymbol{\eta}}=\mathbf{D}(\mathbf{q})+\mathbf{G}(\mathbf{q}, \boldsymbol{\eta}) \mathbf{u}
\end{aligned}
$$

which after rearranging the terms can be brought to the state space form (5).

Suppose now that equations (6) can still be integrated. The result of this integration is a set of $n-m$ holonomic equations, prescribing $n-m$ degrees of freedom in terms of the remaining $m$ :

$$
\mathbf{g}\left(\mathbf{q}_{1}, \mathbf{q}_{2}\right)=\mathbf{0}
$$

The implicit function theorem provides the necessary conditions under which the above equation can be solved for $\mathbf{q}_{1}$ to yield:

$$
\mathbf{q}_{1}=\mathbf{h}\left(\mathbf{q}_{2}\right)
$$

The above relation can be used to eliminate $\mathbf{q}_{1}$ from equation (2b) so as to obtain a reduced order model.

$$
\hat{\mathbf{M}}\left(\mathbf{q}_{2}\right) \ddot{\mathbf{q}}_{2}+\hat{\mathbf{C}}\left(\mathbf{q}_{2}, \dot{\mathbf{q}}_{2}\right) \dot{\mathbf{q}}_{2}+\hat{\mathbf{K}}\left(\mathbf{q}_{2}\right)=\hat{\mathbf{B}}\left(\mathbf{q}_{2}\right) \mathbf{u}, \quad \mathbf{q}_{2} \in \mathbf{Q}_{2} \subset \mathfrak{R}^{m}
$$

Another case is when the dynamic constraints that form equation ( $2 a$ ) are a collection of second order nonholonomic, first order nonholonomic and even holonomic. In this occasion, the procedure outlined for each case should be followed for the part that falls within each category. This could be quite troublesome but it is the only way to clear out the scene and obtain a consistent, minimum order model.

\section{Controllability Issues}

When $k$ dynamic constraints are holonomic, then the system motion is restricted to an $n-k$ dimensional manifold. It cannot reach any position outside this manifold. Points that do not belong to that manifold are simply unreachable.

In the case of completely first order nonholonomic constraints, the configuration space is not confined. The system is known to be accessible. Due, however, to the drift term $\mathbf{D}(\mathbf{q})$ in equation (9), accessibility for the system does not imply control- 
lability. This does not mean that the system is not controllable; it simply means that there is no definite clue that it is. For nonholonomic systems with drift, there is no available general necessary and sufficient result for establishing complete controllability [12]. One has to resort to other forms of controllability, such as strong accessibility and small-time-local controllability. For the latter, there exist only sufficient conditions, but once it has been established one can use the manifold of equilibrium points of the drift vector field to reach an arbitrary small neighborhood of the desired configuration. A difficult point is that first order nonholonomic systems are not stabilizable via continuous time-invariant state feedback. In summary, this is a situation that one should wish to avoid.

If however the system (5) is proved to be second-order nonholonomic, it has been proved [10] that it is automatically strongly accessible. Moreover, there is even a chance for smooth feedback stabilization, provided that a sufficient condition for non-existence of a smooth stabilizing control law is not satisfied:

Theorem 1 [10]: Assume that $R_{i}(\mathbf{x}, \mathbf{0})=0, \quad \forall \mathbf{x} \in \mathbf{M}$, for $i=1, \ldots, n-m$. Let $n-m \geq 1$ and let $\left(\mathbf{x}^{e}, \mathbf{0}\right)$ denote an equilibrium solution. Then the second order nonholonomic system (5) is not asymptotically stabilizable to $\left(\mathbf{x}^{e}, \mathbf{0}\right)$, using time-invariant continuous (static or dynamic) state feedback law.

If on the other hand $\forall i, \exists \mathbf{x} \in \mathbf{M} \mid R_{i}(\mathbf{x}, 0) \neq 0$, then the system could perhaps be stabilizable by continuous control law.

\subsection{The Finite Element Model}

Lets return now to the finite element model we have developed for the object. The finite element analysis results to a dynamic model which is linear [13]:

$$
\mathbf{M} \ddot{\mathbf{q}}+\mathbf{C} \dot{\mathbf{q}}+\mathbf{K} \mathbf{q}=\mathbf{B} \mathbf{u}
$$

Moreover, the characteristic matrices $\mathbf{M}, \mathbf{C}, \mathbf{K}$ and $\mathbf{B}$ are independent of the node coordinates. The finite element method provides a meaningful way of linearizing the original dynamic equations of the deformable object. Under specific conditions for selecting the interpolation functions within the element, the linear model can be proved to be equivalent to the initial nonlinear differential equation, so that none of the information contained in the original equations is lost in the process and all modes are represented by the approximated model.

The state equations derived from (10) have the form

$$
\begin{gathered}
\ddot{\mathbf{q}}_{2}=\mathbf{v} \\
\ddot{\mathbf{q}}_{1}=\mathbf{J} \ddot{\mathbf{q}}_{2}+\mathbf{R}(\mathbf{q}, \dot{\mathbf{q}})
\end{gathered}
$$

The state equations can be formed as 


$$
\begin{aligned}
& \dot{\mathbf{x}}_{1}=\mathbf{x}_{3} \\
& \dot{\mathbf{x}}_{2}=\mathbf{x}_{4} \\
& \dot{\mathbf{x}}_{3}=\mathbf{J} \mathbf{v}+\mathbf{R}\left(\mathbf{x}_{1}, \mathbf{x}_{2}, \mathbf{x}_{3}, \mathbf{x}_{4}\right) \\
& \dot{\mathbf{x}}_{4}=\mathbf{v}
\end{aligned}
$$

We give the following Lemma:

Lemma 1 : For the system (11) it holds:

1. $\left[\tau_{k}, a d_{\tau_{0}}^{r} \tau_{j}\right\rfloor=0, \quad \forall r \geq 0, \quad k, j \in\{1, \ldots, m\}$

2. $a d_{\tau_{0}}^{1+r} \tau_{j}=(-1)^{r-1}\left[\begin{array}{c}A_{r} \cdot\left[\begin{array}{cc}\mathbf{J}^{T}{ }_{j} & \mathbf{e}^{T}{ }_{j}\end{array}\right]^{T} \\ \mathbf{0}_{m \times 1} \\ B_{r} \cdot\left[\begin{array}{cc}\mathbf{J}^{T}{ }_{j} & \mathbf{e}^{T}{ }_{j}\end{array}\right]^{T} \\ \mathbf{0}_{(m+1) \times 1}\end{array}\right]$ for $r>0, A_{r}, B_{r} \in \Re^{(n-m) \times 1}, \mathbf{J}_{j}$ the $j^{\text {th }}$ column of $\mathbf{J}$ and $\mathbf{e}_{j}$ is the $j^{\text {th }}$ base vector of $\Re^{m}$

3. The vectors that form the vector field $a d_{\tau_{0}}^{1+r} \tau_{j}$ can be expressed as follows:

$$
\begin{aligned}
B_{r}=\frac{\partial \mathbf{R}}{\partial \mathbf{x}_{1}} A_{r-1}+\frac{\partial \mathbf{R}}{\partial \dot{\mathbf{x}}_{1}} B_{r-1} \text { with }^{B_{1}}=\frac{\partial \mathbf{R}}{\partial \mathbf{x}}+\frac{\partial \mathbf{R}}{\partial \dot{\mathbf{x}}_{1}} \cdot \frac{\partial \mathbf{R}}{\partial \dot{\mathbf{x}}} \\
A_{r}=B_{r-1} \\
A_{1}=\frac{\partial \mathbf{R}}{\partial \dot{\mathbf{x}}}
\end{aligned}
$$

\section{Proof:}

1. Define $\overline{\mathbf{x}}=\left[\begin{array}{lll}\mathbf{x} & \dot{\mathbf{x}} & t\end{array}\right]$. For the Lie bracket it is $\left[\tau_{k}, a d_{\tau_{0}}^{r} \tau_{j}\right]=\left[\tau_{k},\left[\tau_{0}, a d_{\tau_{0}}^{r-1}\right]\right] . \quad$ For $\quad r=0 \quad$ we have $\left[\tau_{k}, \tau_{j}\right]=\frac{\partial \tau_{j}}{\partial \overline{\mathbf{x}}} \tau_{k}-\frac{\partial \tau_{k}}{\partial \overline{\mathbf{x}}} \tau_{j}$. Recalling Definition 1, and calculating $\tau_{j}$ for the system (11), we can see that $\tau_{j}=\left[\begin{array}{c}\mathbf{0}_{n \times 1} \\ \mathbf{J}_{j} \\ \mathbf{e}_{j} \\ 0\end{array}\right]$. However $\mathbf{J}$ is independent of $\overline{\mathbf{x}}$, so that both $\frac{\partial \tau_{j}}{\partial \overline{\mathbf{x}}}$ and $\frac{\partial \tau_{k}}{\partial \overline{\mathbf{x}}}$ are zero. It is easy to see that any Lie bracket $\left[\tau_{k}, \mathbf{0}\right]=\mathbf{0}$. 
2. and 3. It can be shown by induction: We first verify for $r=1$. It is $a d_{\tau_{0}}^{2} \tau_{j}=\left[\tau_{0},\left[\tau_{0}, \tau_{j}\right]\right]$. The inner bracket is shown to be: $\left[\tau_{0}, \tau_{j}\right]=-\frac{\partial \tau_{0}}{\partial \overline{\mathbf{x}}} \tau_{j}$, where

$$
\frac{\partial \tau_{0}}{\partial \overline{\mathbf{x}}}=\left[\begin{array}{ccc}
\left(\frac{\partial \mathbf{R}}{\partial \mathbf{x}}\right)_{(n-m) \times n} & \mathbf{I}_{n \times n} & \mathbf{0}_{n \times 1} \\
& \left(\frac{\partial \mathbf{R}}{\partial \dot{\mathbf{x}}}\right)_{(n-m) \times n} & \mathbf{0}_{(n-m) \times 1} \\
\mathbf{0}_{(m+1) \times(2 n+1)} &
\end{array}\right] .
$$

Matrix I is the identity matrix. Now, for $r=1$, $a d_{\tau_{0}}^{2} \tau_{j}=\frac{\partial\left[\tau_{0}, \tau_{j}\right]}{\partial \overline{\mathbf{x}}} \tau_{0}-\frac{\partial \tau_{0}}{\partial \overline{\mathbf{x}}}\left[\tau_{0}, \tau_{j}\right]$. By the structure of the finite element model (10), it can be seen that $\mathbf{R}$ does not contain quadratic terms in $\mathbf{x}$, nor in $\dot{\mathbf{x}}$. Therefore, $\frac{\partial \tau_{0}}{\partial \overline{\mathbf{x}}}$ is independent of $\overline{\mathbf{x}}$. On the other hand, $\tau_{j}$ is also constant since $\mathbf{J}$ is independent of $\overline{\mathbf{x}}$.

Thus $\frac{\partial\left[\tau_{0}, \tau_{j}\right]}{\partial \overline{\mathbf{x}}}=0$ and $a d_{\tau_{0}}^{2} \tau_{j}=-\frac{\partial \tau_{0}}{\partial \overline{\mathbf{x}}}\left[\tau_{0}, \tau_{j}\right]=\left(\frac{\partial \tau_{0}}{\partial \overline{\mathbf{x}}}\right)^{2} \tau_{j}$. Calculating the square of this matrix one can verify that

$$
\begin{aligned}
& \left(\frac{\partial \tau_{0}}{\partial \overline{\mathbf{x}}}\right)^{2}= \\
& {\left[\begin{array}{cc}
\frac{\partial \mathbf{R}}{\partial \mathbf{x}_{1}} & \frac{\partial \mathbf{R}}{\partial \mathbf{x}_{2}} \\
\mathbf{0}_{m \times(n-m)} & \mathbf{0}_{m \times m}
\end{array}\right)_{n \times n}} \\
& \left(\begin{array}{cc}
\frac{\partial \mathbf{R}}{\partial \dot{\mathbf{x}}_{\mathbf{1}}} & \frac{\partial \mathbf{R}}{\partial \dot{\mathbf{x}}_{2}} \\
\mathbf{0}_{m \times(n-m)} & \mathbf{0}_{m \times m}
\end{array}\right)_{n \times n} \\
& \left(\begin{array}{cc}
\left(\frac{\partial \mathbf{R}}{\partial \dot{\mathbf{x}}_{\mathbf{1}}}\right)^{2} & \frac{\partial \mathbf{R}}{\partial \dot{\mathbf{x}}_{1}} \cdot \frac{\partial \mathbf{R}}{\partial \mathbf{x}_{2}} \\
\mathbf{0}_{m \times(n-m)} & \mathbf{0}_{m \times m}
\end{array}\right)_{n \times n} \\
& \mathbf{0}_{1 \times n} \\
& \left(\begin{array}{c}
\frac{\partial \mathbf{R}}{\partial \mathbf{x}} \\
\mathbf{0}_{m \times n}
\end{array}\right)_{n \times n}+\left(\begin{array}{cc}
\left(\frac{\partial \mathbf{R}}{\partial \dot{\mathbf{x}}_{\mathbf{1}}}\right)^{2} & \frac{\partial \mathbf{R}}{\partial \dot{\mathbf{x}}_{1}} \cdot \frac{\partial \mathbf{R}}{\partial \dot{\mathbf{x}}_{2}} \\
\mathbf{0}_{m \times(n-m)} & \mathbf{0}_{m \times m}
\end{array}\right)_{n \times n} \\
& \mathbf{0}_{1 \times n}
\end{aligned}
$$

which multiplied by $\tau_{j}$ yields: 


$$
a d_{\tau_{0}}^{2} \tau_{j}=\left[\begin{array}{c}
\frac{\partial \mathbf{R}}{\partial \dot{\mathbf{x}}} \cdot\left[\begin{array}{c}
\mathbf{J}_{j} \\
\mathbf{e}_{j}
\end{array}\right] \\
\mathbf{0}_{m \times 1} \\
\left.\left(\frac{\partial \mathbf{R}}{\partial \mathbf{x}}+\left(\left(\frac{\partial \mathbf{R}}{\partial \dot{\mathbf{x}}_{1}}\right)^{2} \frac{\partial \mathbf{R}}{\partial \dot{\mathbf{x}}_{1}} \cdot \frac{\partial \mathbf{R}}{\partial \dot{\mathbf{x}}_{2}}\right)\right) \cdot \cdot \cdot \mathbf{J}_{j}\right] \mathbf{e}_{j}
\end{array}\right]=\left[\begin{array}{c}
\frac{\partial \mathbf{R}}{\partial \dot{\mathbf{x}}} \cdot\left[\begin{array}{c}
\mathbf{J}_{j} \\
\mathbf{e}_{j}
\end{array}\right] \\
\mathbf{0}_{(m+1) \times 1}
\end{array}\right]
$$

This can be written equivalently

$$
a d_{\tau_{0}}^{2} \tau_{j}=\left[\begin{array}{c}
\frac{\partial \mathbf{R}}{\partial \dot{\mathbf{x}}} \cdot\left[\begin{array}{c}
\mathbf{J}_{j} \\
\mathbf{e}_{j}
\end{array}\right] \\
\mathbf{0}_{m \times 1} \\
\left(\frac{\partial \mathbf{R}}{\partial \mathbf{x}}+\frac{\partial \mathbf{R}}{\partial \dot{\mathbf{x}}_{1}} \cdot \frac{\partial \mathbf{R}}{\partial \dot{\mathbf{x}}}\right) \cdot\left[\begin{array}{l}
\mathbf{J}_{j} \\
\mathbf{e}_{j}
\end{array}\right] \\
\mathbf{0}_{(m+1) \times 1}
\end{array}\right],
$$

which proves our result for $r=1$.

Suggest now that it holds for $r=k$. Then for $r=k+1$,

$$
a d_{\tau_{0}}^{1+(k+1)} \tau_{j}=\frac{\partial a d_{\tau_{0}}^{1+k} \tau_{j}}{\partial \overline{\mathbf{x}}} \tau_{0}-\frac{\partial \tau_{0}}{\partial \overline{\mathbf{x}}} a d_{\tau_{0}}^{1+k} \tau_{j}=-\frac{\partial \tau_{0}}{\partial \overline{\mathbf{x}}} a d_{\tau_{0}}^{1+k} \tau_{j},
$$

equivalently,

$$
\begin{aligned}
& a d_{\tau_{0}}^{1+(k+1)} \tau_{j}=-\left[\begin{array}{ccc}
\mathbf{0}_{n \times n} & \mathbf{I}_{n \times n} & \mathbf{0}_{n \times 1} \\
\left(\frac{\mathbf{R}}{\partial \mathbf{x}}\right)_{(n-m) \times n} & \left(\frac{\partial \mathbf{R}}{\partial \dot{\mathbf{x}}}\right)_{(n-m) \times n} & \mathbf{0}_{(n-m) \times 1} \\
\mathbf{0}_{(m+1) \times(2 n+1)} &
\end{array}\right] \cdot(-1)^{k-1}\left[\begin{array}{c}
A_{k} \cdot\left[\begin{array}{l}
\mathbf{J}_{j} \\
\mathbf{e}_{j}
\end{array}\right] \\
\mathbf{0}_{m \times 1} \\
B_{k} \cdot\left[\begin{array}{l}
\mathbf{J}_{j} \\
\mathbf{e}_{j}
\end{array}\right] \\
\mathbf{0}_{(m+1) \times 1}
\end{array}\right]= \\
& =(-1)^{(k+1)-1}\left[\begin{array}{c}
B_{k} \cdot\left[\begin{array}{c}
\mathbf{J}_{j} \\
\mathbf{e}_{j}
\end{array}\right] \\
\frac{\partial \mathbf{R}}{\partial \mathbf{x}_{1}} A_{k} \cdot\left[\begin{array}{c}
\mathbf{J}_{j} \\
\mathbf{e}_{j \times 1}
\end{array}\right]+\frac{\partial \mathbf{R}}{\partial \dot{\mathbf{x}}_{1}} B_{k} \cdot\left[\begin{array}{l}
\mathbf{J}_{j} \\
\mathbf{e}_{j}
\end{array}\right]
\end{array}\right]
\end{aligned}
$$

which completes the proof.

We are now ready to present our main result:

Proposition 1: Define the sequence of matrices: 


$$
\left[\begin{array}{c}
A_{1} \\
\mathbf{0}_{m \times 1} \\
B_{1} \\
\mathbf{0}_{(m+1) \times 1}
\end{array}\right] \cdot\left[\begin{array}{c}
\mathbf{J} \\
\mathbf{I}_{m \times m}
\end{array}\right] \cdots\left[\begin{array}{c}
A_{r} \\
\mathbf{0}_{m \times 1} \\
B_{r} \\
\mathbf{0}_{(m+1) \times 1}
\end{array}\right] \cdot\left[\begin{array}{c}
\mathbf{J} \\
\mathbf{I}_{m \times m}
\end{array}\right] \cdots
$$

where $A_{1}, B_{1}, A_{r}, B_{r}$ are

$$
\begin{aligned}
B_{1} & =\frac{\partial \mathbf{R}}{\partial \mathbf{x}}+\frac{\partial \mathbf{R}}{\partial \dot{\mathbf{x}}_{1}} \cdot \frac{\partial \mathbf{R}}{\partial \dot{\mathbf{x}}}, & B_{r} & =\frac{\partial \mathbf{R}}{\partial \mathbf{x}_{1}} A_{r-1}+\frac{\partial \mathbf{R}}{\partial \dot{\mathbf{x}}_{1}} B_{r-1} . \\
A_{1} & =\frac{\partial \mathbf{R}}{\partial \dot{\mathbf{x}}} & A_{r} & =B_{r-1}
\end{aligned}
$$

If for some $r$, the extended matrix

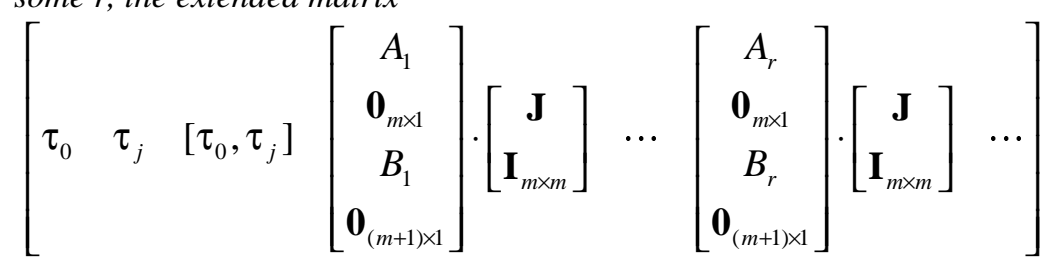

has rank $2 n+1$ then the system (11) is second order nonholonomic.

Proof: The proof follows from the Definition 1 and the previous Lemma. The series defined above is directly associated with the accessibility distribution. Indeed the vector fields $\tau_{0}, \tau_{j},\left[\tau_{0}, \tau_{j}\right], a d_{\tau_{0}}^{1+r} \tau_{j}$ define one Phillip Hall basis for the accessibility distribution. It is easily shown that vector fields $\tau_{0}, \tau_{j},\left[\tau_{0}, \tau_{j}\right]$ are linearly independent. This is obvious for $\tau_{0}, \tau_{j}$ by their definition. On the other hand,

$$
\left[\tau_{0}, \tau_{j}\right]=-\left[\begin{array}{ccc}
\mathbf{0}_{n \times n} & \mathbf{I}_{n \times n} & \mathbf{0}_{n \times 1} \\
\left(\frac{\partial \mathbf{R}}{\partial \mathbf{x}}\right)_{(n-m) \times n} & \left(\frac{\partial \mathbf{R}}{\partial \dot{\mathbf{x}}}\right)_{(n-m) \times n} & \mathbf{0}_{(n-m) \times 1} \\
\mathbf{0}_{(m+1) \times(2 n+1)} &
\end{array}\right] \cdot\left[\begin{array}{c}
\mathbf{0}_{n \times 1} \\
\mathbf{J}_{j} \\
\mathbf{e}_{j} \\
0
\end{array}\right]=-\left[\begin{array}{c}
\mathbf{J}_{j} \\
\mathbf{e}_{j} \\
* \\
0
\end{array}\right]
$$

As it can be seen by inspection, the $2 m+1$ vector fields $\tau_{0}, \tau_{j},\left[\tau_{0}, \tau_{j}\right]$ are linearly independent and generate a $2 m+1$ dimensional distribution. Including more vector fields $a d_{\tau_{0}}^{r+1} \tau_{j}$ in the set, the dimension of the distribution grows. This sequence of distributions $G_{i}=G_{i-1}+\left[G_{1}, G_{i-1}\right]$ is a filtration. Each $G_{i}$ is spanned by vector fields of the previous one plus some vector fields formed by taking $i-1$ Lie brackets. If for some $\mathrm{r}$, the rank of the extended matrix is $2 \mathrm{n}+1$ is means that it contains $2 n+1$ linearly independent columns. The columns of the matrix, however, are exactly the vector fields that one would calculate for the P. Hall 
basis of the accessibility algebra. Choosing $2 n+1$ linearly independent columns one has $2 n+1$ independent vector fields that span the accessibility algebra of the system (11). By Definition 1, (11) is second order nonholonomic.

Generally, as it will be shown in the examples, systems derived by finite elements are usually second order nonholonomic. The process of determining the existence of second order nonholonomic constraints in systems with possibly hundred degrees of freedom through the conventional way of seeking for a Phillip Hall basis becomes awfully cumbersome. On the other hand, the algorithm just described provides an immediate way of investigation since one can easily automate the above procedure.

Being second order nonholonomic, the finite element model (11) is strongly accessible. One can easily verify however, that it does not satisfy the sufficient condition for small time local controllability, presented in [14]. However, this does not rule out the possibility that the system may be small time locally controllable. In fact, since the finite element model is linear, a simple matrix calculation can show whether that system is controllable. In that case the stabilizing feedback law is not only continuous but also linear! This comes in accordance to Theorem 1, since the

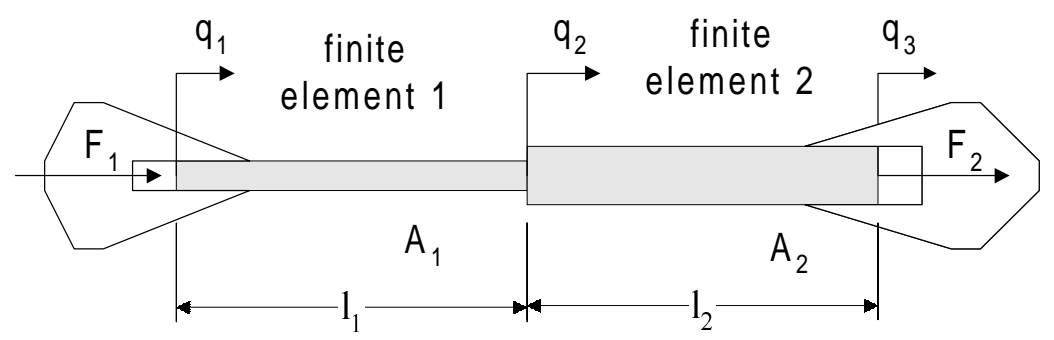

Figure 1: A deformable object under axial load

condition for nonexistence of a smooth feedback law, $\mathbf{R}\left(\mathbf{x}^{e}, \mathbf{0}\right)=\mathbf{0}, \quad \forall \mathbf{x}$, is not satisfied.

\section{Example - Rod Under Axial Load}

Consider a beam under axial load (Figure 1). The beam is divided into two finite elements. The system has three degrees of freedom, two of which are directly controlled. The element characteristic matrices are:

$$
\mathbf{M}=\frac{\rho A \ell}{6}\left[\begin{array}{ll}
2 & 1 \\
1 & 2
\end{array}\right], \quad \mathbf{C}=\frac{\mu A \ell}{6}\left[\begin{array}{ll}
2 & 1 \\
1 & 2
\end{array}\right], \quad \mathbf{K}=\frac{A E}{\ell}\left[\begin{array}{cc}
1 & -1 \\
-1 & 1
\end{array}\right]
$$

where $\rho$ is the density of the material, $A$ is the cross section, $\ell$ is the length of the element and $E$ the elasticity modulus. The equations for the two elements are 
assembled and after rearranging the terms to distinguish the actuated part from the unactuated, the complete equations have the following form:

$$
\begin{gathered}
{\left[\begin{array}{ccc}
\frac{\rho\left(A_{1} \ell_{1}+A_{2} \ell_{2}\right)}{3} & \frac{\rho A_{1} \ell_{1}}{6} & \frac{\rho A_{2} \ell_{2}}{6} \\
\frac{\rho A_{1} \ell_{1}}{6} & \frac{\rho A_{1} \ell_{1}}{3} & 0 \\
\frac{\rho A_{2} \ell_{2}}{6} & 0 & \frac{\rho A_{2} \ell_{2}}{3}
\end{array}\right] \cdot\left[\begin{array}{l}
\ddot{q}_{2} \\
\ddot{q}_{1} \\
\ddot{q}_{3}
\end{array}\right]+\left[\begin{array}{ccc}
\frac{\mu\left(A_{1} \ell_{1}+A_{2} \ell_{2}\right)}{3} & \frac{\mu A_{1} \ell_{1}}{6} & \frac{\mu A_{2} \ell_{2}}{6} \\
\frac{\mu A_{1} \ell_{1}}{6} & \frac{\mu A_{1} \ell_{1}}{3} & 0 \\
\frac{\mu A_{2} \ell_{2}}{6} & 0 & \frac{\mu A_{2} \ell_{2}}{3}
\end{array}\right] \cdot\left[\begin{array}{l}
\dot{q}_{2} \\
\dot{q}_{1} \\
\dot{q}_{3}
\end{array}\right]} \\
+\left[\begin{array}{ccc}
\frac{A_{1} E}{\ell_{1}}+\frac{A_{2} E}{\ell_{2}} & -\frac{A_{1} E}{\ell_{1}} & -\frac{A_{2} E}{\ell_{2}} \\
-\frac{A_{1} E}{\ell_{1}} & \frac{A_{1} E}{\ell_{1}} & 0 \\
-\frac{A_{2} E}{\ell_{2}} & 0 & \frac{A_{2} E}{\ell_{2}}
\end{array}\right] \cdot\left[\begin{array}{c}
q_{2} \\
q_{1} \\
q_{3}
\end{array}\right]=\left[\begin{array}{c}
0 \\
F_{1} \\
F_{2}
\end{array}\right]
\end{gathered}
$$

Applying the linearizing feedback (3) the above equations become

$$
\begin{aligned}
& \ddot{q}_{1}=v_{1} \\
& \ddot{q}_{3}=v_{2} \\
& \ddot{q}_{2}=\mathbf{J}\left[\begin{array}{ll}
v_{1} & v_{2}
\end{array}\right]^{T}+R
\end{aligned}
$$

where

$$
\begin{aligned}
\mathbf{J}=- & \frac{1}{2\left(A_{1} \ell_{1}+A_{2} \ell_{2}\right)}\left[\begin{array}{ll}
A_{1} \ell_{1} & A_{2} \ell_{2}
\end{array}\right] \\
R=- & \frac{3}{\rho\left(A_{1} \ell_{1}+A_{2} \ell_{2}\right)}\left[\frac{\mu}{3}\left(A_{1} \ell_{1}+A_{2} \ell_{2}\right) \dot{q}_{2}+\frac{\mu A_{1} \ell_{1}}{6} \dot{q}_{1}+\frac{\mu A_{2} \ell_{2}}{6} \dot{q}_{3}\right. \\
& \left.+E\left(\frac{A_{1}}{\ell_{1}}+\frac{A_{2}}{\ell_{2}}\right) q_{2}-\frac{A_{1} E}{\ell_{1}} q_{1}-\frac{A_{2} E}{\ell_{2}} q_{3}\right]
\end{aligned}
$$

As it can be verified, the linear system is controllable which means that it can be driven with continuous linear feedback law. Indeed, condition $\mathbf{R}\left(\mathbf{q}^{e}, \mathbf{0}\right)=\mathbf{0}, \quad \forall \mathbf{q}$ does not hold.

Set $x_{1}=q_{2}, \mathbf{x}_{2}=\left[\begin{array}{ll}q_{1} & q_{2}\end{array}\right]^{T}, x_{3}=\dot{x}_{1}, \mathbf{x}_{4}=\dot{\mathbf{x}}_{2}$

Using Proposition 1 we can prove that the system is second order nonholonomic. Notice how the columns in the sequence of matrices defined in Proposition 1 give exactly the vector fields which were to be calculated for the P. Hall basis.

Lets calculate first the vector fields that are formed with up to one level of bracketing:

$$
\begin{aligned}
\tau_{0} & =\left[\begin{array}{lllllll}
\dot{q}_{2} & \dot{q}_{1} & \dot{q}_{3} & R & 0 & 0 & 1
\end{array}\right]^{T} \\
\tau_{1} & =\left[\begin{array}{llllllll}
0 & 0 & 0 & J_{1} & 1 & 0 & 0
\end{array}\right]^{T} \\
\tau_{2} & =\left[\begin{array}{lllllll}
0 & 0 & 0 & J_{2} & 0 & 1 & 0
\end{array}\right]^{T}
\end{aligned}
$$

and continuing with one level of bracketing: 


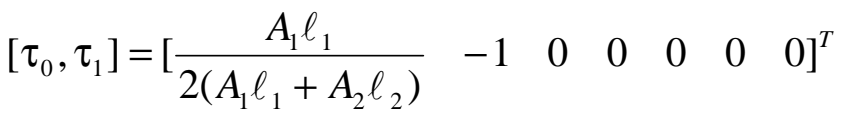

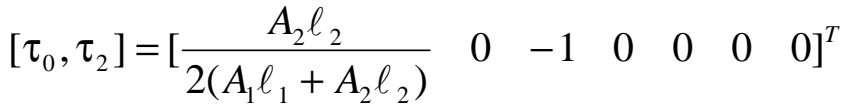

Continuing to higher levels yields:

$$
\begin{aligned}
& a d_{\tau_{0}}^{2} \tau_{1}=\left[\begin{array}{lllllll}
0 & 0 & 0 & 0 & \frac{3 A_{1} E\left(3 A_{1} \ell_{1} \ell_{2}+2 A_{2} \ell_{2}{ }^{2}+A_{2} \ell_{1}{ }^{2}\right)}{2 \rho\left(A_{1} \ell_{1}+A_{2} \ell_{2}\right)^{2} \ell_{1} \ell_{2}} & 0 & 0
\end{array}\right]^{T} \\
& a d_{\tau_{0}}^{2} \tau_{2}=\left[\begin{array}{lllllllll}
0 & 0 & 0 & 0 & \frac{3 A_{2} E\left(3 A_{2} \ell_{1} \ell_{2}+2 A_{1} \ell_{1}{ }^{2}+A_{1} \ell_{2}{ }^{2}\right)}{2 \rho\left(A_{1} \ell_{1}+A_{2} \ell_{2}\right)^{2} \ell_{1} \ell_{2}} & 0 & 0
\end{array}\right]^{T} \\
& a d_{\tau_{0}}^{3} \tau_{1}=\frac{3 A_{1} E\left(3 A_{1} \ell_{1} \ell_{2}+2 A_{2} \ell_{2}{ }^{2}+A_{2} \ell_{1}{ }^{2}\right)}{\rho\left(A_{1} \ell_{1}+A_{2} \ell_{2}\right)^{2} \ell_{1} \ell_{2}}\left[\begin{array}{lllllll}
0 & -\frac{1}{2} & 0 & 0 & \frac{\mu}{2 \rho} & 0 & 0
\end{array}\right]^{T} \\
& a d_{\tau_{0}}^{3} \tau_{1}=\frac{3 A_{2} E\left(3 A_{2} \ell_{1} \ell_{2}+2 A_{1} \ell_{1}{ }^{2}+A_{1} \ell_{2}{ }^{2}\right)}{\rho\left(A_{1} \ell_{1}+A_{2} \ell_{2}\right)^{2} \ell_{1} \ell_{2}}\left[\begin{array}{lllllll}
0 & -\frac{1}{2} & 0 & 0 & \frac{\mu}{2 \rho} & 0 & 0
\end{array}\right]^{T}
\end{aligned}
$$

Since vector fields $\tau_{0}, \tau_{j},\left[\tau_{0}, \tau_{j}\right], a d_{\tau_{0}}^{2} \tau_{1}, a d_{\tau_{0}}^{3} \tau_{1}$ are independent, the system is second order nonholonomic. Now, notice how these vector fields appear explicitly in the extended matrix of Proposition 1: 


$$
\begin{aligned}
& L_{1}=\left[\begin{array}{c}
\frac{\partial R}{\partial \dot{\mathbf{x}}} \\
0 \\
\frac{\partial R}{\partial \mathbf{x}}+\frac{\partial R}{\partial \dot{\mathbf{x}}_{2}} \cdot \frac{\partial R}{\partial \dot{\mathbf{x}}} \\
0 \\
0
\end{array}\right] \cdot\left[\begin{array}{cc}
J_{1} & J_{2} \\
1 & 0 \\
0 & 1
\end{array}\right]= \\
& =\left[\begin{array}{cc}
0 & 0 \\
0 & 0 \\
0 & 0 \\
\frac{3 A_{1} E\left(3 A_{1} \ell_{1} \ell_{2}+A_{2} \ell_{1}^{2}+2 A_{2} \ell_{2}{ }^{2}\right)}{2 \rho \ell_{1} \ell_{2}\left(A_{1} \ell_{1}+A_{2} \ell_{2}\right)^{2}} & \frac{3 A_{2} E\left(3 A_{2} \ell_{1} \ell_{2}+A_{1} \ell_{2}^{2}+2 A_{1} \ell_{1}^{2}\right)}{2 \rho \ell_{1} \ell_{2}\left(A_{1} \ell_{1}+A_{2} \ell_{2}\right)^{2}} \\
0 & 0 \\
0 & 0 \\
0 & 0
\end{array}\right] \\
& L_{2}=-\left[\begin{array}{c}
A_{2} \\
0 \\
0 \\
B_{2} \\
0 \\
0 \\
0
\end{array}\right] \cdot\left[\begin{array}{cc}
J_{1} & J_{2} \\
1 & 0 \\
0 & 1
\end{array}\right]= \\
& =\left[\begin{array}{cc}
-\frac{3 A_{1} E\left(3 A_{1} \ell_{1} \ell_{2}+A_{2} \ell_{1}^{2}+2 A_{2} \ell_{2}^{2}\right)}{2 \rho \ell_{1} \ell_{2}\left(A_{1} \ell_{1}+A_{2} \ell_{2}\right)} & -\frac{3 A_{2} E\left(3 A_{2} \ell_{1} \ell_{2}+A_{1} \ell_{2}{ }^{2}+2 A_{1} \ell_{1}^{2}\right)}{2 \rho \ell_{1} \ell_{2}\left(A_{1} \ell_{1}+A_{2} \ell_{2}\right)} \\
0 & 0 \\
0 & 0 \\
\frac{3 \mu A_{1} E\left(3 A_{1} \ell_{1} \ell_{2}+A_{2} \ell_{1}^{2}+2 A_{2} \ell_{2}^{2}\right)}{\rho^{2} \ell_{1} \ell_{2}\left(A_{1} \ell_{1}+A_{2} \ell_{2}\right)^{2}} & \frac{3 \mu A_{2} E\left(3 A_{2} \ell_{1} \ell_{2}+A_{1} \ell_{2}^{2}+2 A_{1} \ell_{1}^{2}\right)}{\rho^{2} \ell_{1} \ell_{2}\left(A_{1} \ell_{1}+A_{2} \ell_{2}\right)^{2}} \\
0 & 0 \\
0 & 0 \\
0 & 0
\end{array}\right]
\end{aligned}
$$

In this case the partial derivatives of $\mathrm{R}$ are calculated only once, at the beginning of the procedure, whereas the direct calculation of the Lie brackets of the vector fields requires the calculation of the Jacobians of the vector fields involved. 


\section{Material Constraints}

The degrees of freedom of the manipulated object may be subject to constraints. These constraints can arise from material strength limitations and/or obstacle avoidance requirements. While the latter apply directly on the object degrees of freedom, the former are usually expressed in the form

$$
\boldsymbol{\sigma} \leq \overline{\boldsymbol{\sigma}}
$$

where $\boldsymbol{\sigma}$ is the stress tensor of the structure and $\overline{\boldsymbol{\sigma}}$ is the maximum admissible stress specified for the particular material and object. With some algebraic manipulation the above relation can be translated in terms of the finite element model node displacements. If one recalls the well known expressions that relate the deformation $\boldsymbol{\varepsilon}$, the displacement $\mathbf{U}$, and the stress $\boldsymbol{\sigma}$,

$$
\boldsymbol{\varepsilon}=\mathbf{D} \cdot \mathbf{U}, \quad \boldsymbol{\sigma}=\mathbf{E} \cdot \boldsymbol{\varepsilon}
$$

along with the element interpolation functions

$$
\mathbf{U}=\mathbf{N} \cdot \mathbf{q}
$$

the material constraints can be expressed in terms of the degrees of freedom. These constraints can be included in (10) with the use of Kuhn-Tucker multipliers:

$$
\begin{aligned}
& \mathbf{M} \ddot{\mathbf{q}}+\mathbf{C} \dot{\mathbf{q}}+\mathbf{K} \mathbf{q}=\mathbf{B} \mathbf{u}+\left(\frac{\partial \mathbf{r}}{\partial \mathbf{q}}\right)^{T} \boldsymbol{\mu} \\
& \boldsymbol{\mu}^{T} \mathbf{r}(\mathbf{q})=0, \quad \boldsymbol{\mu} \geq 0 .
\end{aligned}
$$

In this case, the multipliers cannot be eliminated, because they correspond to inequality conditions which do not reduce the dimension of the state space. The constraint terms in the above equations can be included, though, in the elastic and gravity forces terms as follows:

$$
\begin{aligned}
& \mathbf{m}_{11} \ddot{\mathbf{q}}_{1}+\mathbf{m}_{12} \ddot{\mathbf{q}}_{2}+\mathbf{c}_{1} \dot{\mathbf{q}}+\mathbf{k}_{1}(\mathbf{q}, \boldsymbol{\mu})=0 \\
& \mathbf{m}_{21} \ddot{\mathbf{q}}_{1}+\mathbf{m}_{22} \ddot{\mathbf{q}}_{2}+\mathbf{c}_{2} \dot{\mathbf{q}}+\mathbf{k}_{2}(\mathbf{q}, \boldsymbol{\mu})=\mathbf{b}(\mathbf{u}) \\
& \boldsymbol{\mu}^{T} \mathbf{r}(\mathbf{q})=0, \quad \boldsymbol{\mu} \geq 0 .
\end{aligned}
$$

When the stress conditions are satisfied, $\boldsymbol{\mu}$ vanishes and the equations describe the motion of the unconstraint system.

It should not be attempted however to determine the existence of nonholonomic constraints using the above equations. One the one hand, when constraints are respected, the equations coincide with (10). If the constraints are violated, it may be too late to examine controllability. On the other hand, material constraints do not correspond to the natural behavior of the system but are rather externally imposed conditions which are stated in order to confine deformations and avoid, perhaps, fracture. They are primarily used for optimization purposes. At any case, the results of the preceding sections may no longer hold if the inclusion of the material constraints destroys the linear structure of the elastic term. 


\section{Conclusion}

A deformable object under manipulation can be modeled using finite element. This way, the distributed parameter model of the original system is converted to a finite dimensional underactuated mechanical system. In this light, the class of objects under study can be extended to include systems with combination of rigid and deformable objects, unactuated joints, flexible links and joints, rolling contacts etc.

This modeling method reveals a set of dynamic constraints. Depending on their type, several conclusions about the behavior of the model under control strategies can be drawn. For each type, the deformable object model is treated accordingly. Apart from these dynamic constraints, however, there could be additional ones that can relate to material strength limitations and/or obstacle avoidance requirements. A way to incorporate these constraints into the object model is described.

The study of deformable objects in the framework of underactuated mechanical systems indicated the existence of second order nonholonomic constraints. For the identification of this type of constraints there exist some heavy mathematical tools from nonlinear control. The model derived from the finite element methodology however, is linear and one should not be obliged to use such complex techniques. For this purpose, an alternative methodology has been developed, which does not require special mathematical skills and knowledge, and is also more computationally efficient than the original method. This method is illustrative with a simple example.

\section{References}

[1] Tanner H G, Kyriakopoulos K J 1998 Modeling of multiple mobile manipulators handling a common deformable object. Journal of Robotic Systems, (15) 11: 599623.

[2] Sun D, Shi X, Liu Y 1996 Modeling and cooperation of multiple mobile manipulators handling a common deformable object. In: Proc. of the 1996 IEEE Int. Conference on Robotics and Automation, Minneapolis, Minnesota, pp. 2346-2351.

[3] Terzopoulos D, Fleischer K, 1988 Deformable Models. The Visual Computer (4):306-331.

[4] Kosuge K, Sakai M, Kanitani K, Yoshida H, Fukuda T 1995 Manipulation of a flexible object by dual manipulators. In: Proc. of the 1995 IEEE Int. Conference on Robotics and Automation, pp. 318-322.

[5] Wu J, Luo Z, Yamakita M, Ito K 1996 Adaptive hybrid control of manipulators on uncertain flexible objects. Advanced Robotics (10) 5:469-485.

[6] Yukawa T, Uchiyama M, Inooka H 1996 Stability of Control System in Handling of a Flexible Object by Rigid Arm Robots. In: Proc. of the 1996 IEEE Int. Conference on Robotics and Automation, Minneapolis MN, pp. 2332-2338. 
[7] Tanner H G, Kyriakopoulos K J 1999 Analysis of Deformable Handling. In: Proc. of the 1999 IEEE Int. Conference on Robotics and Automation, Detroit, Michigan, pp 2674-2679.

[8] Spong, M W 1998 Underactuated mechanical systems. In: Siciliano B, Valavanis, K P, (eds) 1998 Control Problems in Robotics and Automation, Lecture Notes in Control and Information Science 230, Springer, pp. 135-150.

[9] Boothby M W 1986 An Introduction to Differentiable Manifolds and Riemannian Geometry, $2^{\text {nd }}$ ed. , Academic Press Inc.

[10] Reyhanoglu M, van der Schaft A, McClamroch N H, Kolmanovsky I 1996 Nonlinear control of a class of underactuated systems. In: Proceedings of the $35^{\text {th }}$ IEEE Conference on Decision and Control, Kobe, Japan, pp. 1682-1687.

[11] Campion G, D' Andrea-Novel B, Bastin G 1991 Modeling and state feedback control of nonholonomic mechanical systems. In: Proceedings of the 1991 IEEE Conference on Decision and Control, Brighton, England.

[12] Nijmeiher H, van der Schaft A J 1990 Nonlinear Dynamical Control Systems, Springer-Verlag.

[13] Rao S S 1989 The finite element method in Engineering, Pergamon.

[14] De Luca A, Mattone A, Oriolo G 1996 Dynamic mobility of redundant robots using end-effector commands. In: Proc. of the 1996 Int. Conference on Robotics and Automation, Minneapolis, MN, pp. 1760-1767. 